Cinémas

Revue d'études cinématographiques

Journal of Film Studies

\title{
Le Déplacement et la crise du réel : la socio-sémiotique et la biphobie de Basic Instinct
}

\section{Ki Namaste}

Volume 3, numéro 2-3, printemps 1993

URI : https://id.erudit.org/iderudit/1001200ar

DOI : https://doi.org/10.7202/1001200ar

Aller au sommaire du numéro

Éditeur(s)

Cinémas

ISSN

1181-6945 (imprimé)

1705-6500 (numérique)

Découvrir la revue

Citer cet article

Namaste, K. (1993). Le Déplacement et la crise du réel : la socio-sémiotique et la biphobie de Basic Instinct. Cinémas, 3(2-3), 223-238.

https://doi.org/10.7202/1001200ar

\section{Résumé de l'article}

Cet article vise à étudier le film Basic Instinct du point de vue de sa narration et de sa composition sémiotique, afin de démontrer comment le film relève, à sa base, de la biphobie. À cet égard, la réponse militante des communautés lesbiennes et gaies face au film, qui le décrivent comme " homophobique ", est nettement insuffisante. L'étude démontre qu'une analyse socio-sémiotique est utile pour le développement d'une théorie et d'une pratique de la bisexualité en tant qu'identité possible. 


\title{
Le Déplacement et la crise du réel : la socio-sémiotique et la biphobie de Basic Instinct
}

\section{Ki Namaste}

\begin{abstract}
RÉSUMÉ
Cet article vise à étudier le film Basic Instinct du point de vue de sa narration et de sa composition sémiotique, afin de démontrer comment le film relève, à sa base, de la biphobie. À cet égard, la réponse militante des communautés lesbiennes et gaies face au film, qui le décrivent comme «homophobique», est nettement insuffisante. L'étude démontre qu'une analyse sociosémiotique est utile pour le développement d'une théorie et d'une pratique de la bisexualité en tant qu'identité possible.
\end{abstract}

\begin{abstract}
The article provides a reading of the narrative and semiotic compositions of the film Basic Instinct, in order to demonstrate its implicit biphobia. The lesbian and gay communities' response to the film, denouncing it as "homophobic", is thus read as a misreading. A social semiotic analysis proves useful, then, for the development of critical bisexual theory and activism.
\end{abstract}

Dans le domaine de la théorie dite queer, il y a une prolifération d'analyses de la constitution textuelle de l'homosexualité. Ce champ d'étude, néamoins, néglige la bisexualité. Parmi les quelques références à cette question 1 , Diana Fuss constate que l'opposition hétérosexualité/ 
homosexualité assimile les autres positions d'identification et du désir :

And what gets left out of the inside/outside, heterosexual/ homosexual opposition, an opposition which could at least plausibly be said to secure its seemingly inviolable structure only by assimilating and internalizing other sexualities (bisexuality, transvestism, transsexualism ..) to its own rigid polar logic? (p.

2).

Mon travail prend la question de Fuss comme point de départ. Je veux aborder le film Basic Instinct de Paul Verhoeven (1992) par rapport à ses inscriptions de la bisexualité afin, peut-être, d'apporter un suplément d'information à une théorie et une politique de la sexualité.

Basic Instinct a provoqué beaucoup de réactions dans les communautés gaie, lesbienne, et féministe. L'histoire du film présente 4 femmes qui tuent des hommes, et la réception critique du film note le renversement propre à cette situation : les femmes, les victimes de la violence dans la société occidentale, sont celles qui commettent les actes violents. Le film est dénoncé comme «homophobique» (la haine contre les gais et les lesbiennes) et misogyne. Nathalie Magnan écrit, dans Gai pied (p. 9), que le film a incité les groupes activistes américains Queer Nation et GLADD (Gay and Lesbian Alliance Against Defamation) à s'organiser. Gai pied résume la position de Magnan en disant qu'«il est plus que nécessaire d'alerter Hollywood et l'industrie du cinéma, ici comme ailleurs, sur des pratiques homophobes dont ils sont coutumiers» (p. 9). À l'encontre de cette réception du film, qui le décrit comme «homophobique» ou «misogyne», je considère que Basic Instinct est plutôt un film biphobique. La biphobie est vue ici non seulement dans la présentation des images négatives de la bisexualité, mais aussi dans le fait qu'on continue et persiste à écrire la bisexualité comme une identité impossible.

\section{Théoriser la bisexualité : problèmes de définition}

Avant de contextualiser la présentation de la bisexualité dans Basic Instinct, il faut aborder les définitions contradictoires de la bisexualité. Le problème d'une écriture de la bisexualité n'est pas limité au milieu hollywoodien, c'est un fondement sur lequel on définit la condition bisexuelle. Le Petit Robert définit ainsi un(e) bisexuel(le) :

Qui concerne les deux sexes dans l'individu humain. Tendances bisexuelles. Qui a des relations sexuelles aussi bien avec des 
hommes que des femmes; qui est à la fois hétérosexuel et homosexuel.

Sur le plan historique, le concept de la bisexualité évoque Freud, mais aussi le travail de Wilhelm Fleiss, pour lequel la bisexualité était un fait physique. Comme le note Michael du Plessis ${ }^{2}$, pour Freud la bisexualité était un état psychique plus que physique. Du Plessis explique que dans les Trois essais sur la sexualité, il y avait une confusion pour Freud :

Freud never could decide once and for all if "bisexuality" designated the coincidence of two anatomical sexes in the same body or the conjunction of the two - or more - kinds of desire in the same subject (p. 2).

Si la bisexualité est le site de la conjonction des sexes anatomiques, le corps bisexuel est ainsi le corps hermaphrodite. C'est pour cela que Freud commence son étude, dans les Trois essais, par une référence à l'hermaphrodite tel que présenté par Platon ${ }^{3}$.

Si on retourne au Petit Robert, on retrouve les mêmes problèmes quant à la précision des définitions de la bisexualité. La définition de «bisexuel(le)» circonscrit le mot «bisexué(e)» au niveau de la biologie. On observe que «bisexué(e)» réfère aux choses qui «possède[nt] les deux sexes, qui produi[sent] simultanément ou successivement des gamètes des deux sexes». Et il est important de noter que cette définition continue en faisant référence à l'autogamie («mode de reproduction par union de gamètes provenant du même individu»), au monoïque (une plante «dont les fleurs mâles et les fleurs femelles sont réunies sur le même pied») et à l'hermaphrodite («qui est doté de caractères des deux sexes»). Il y a donc une double instance de la bisexualité qui n'est jamais explicitée ou clarifiée : un état psychique qui rassemble les qualités nommées masculines et féminines dans un individu, et un état physique qui est à la fois masculin et féminin, qui est l'état de l'hermaphrodite, qui est bisexué.

Dans les études féministes, on évoque souvent la bisexualité afin de théoriser le gender. On cite Hélène Cixous ${ }^{4}$ pour qui la femme est bisexuelle (p. 46). Ici, le gender prend la place de la (bi)sexualité. Autrement dit, il y a d'implicite dans ce glissement l'acte de privilégier le gender à la (bi)sexualité. Ainsi on continue à confondre non seulement les définitions de la (bi)sexualité, mais aussi leurs représentations et leurs inscriptions. La substitution des termes de la sexualité pour ceux 
du gender au sein des études féministes suffit à effacer la possibilité d'énoncer une vraie identité (bi)sexuelle 5 .

Si les définitions pré-citées de la bisexualité évoquent de continuelles contradictions, le fait d'articuler une identité bisexuelle positive, réflexive, et possible s'avère un défi stimulant. En empêchant la représentation d'une identité bisexuelle concevable, on reconfirme une conception de la bisexualité qui contourne les réalités de l'expérience bisexuelle. Autrement dit, on écrit la bisexualité d'une manière «biphobique» 6 .

\title{
Mythologies, discours binaires et la biphobie : la crise du sens
}

Plus qu'une décennie après le SIDA, les bisexuel(le)s sont perçu(e)s comme des personnes obsédées par les activités sexuelles. Davantage que les gais et / ou les lesbiennes, la culture occidentale constate que les bisexuel(le)s sont préoccupé(e)s par le sexe. On les cherche partout, parmi les hétérosexuel(le)s et les homosexuel(le)s. Comme le remarque Jan Zita Grover, cette conceptualisation des bisexuel(le)s est indissociable des discours dominants sur le SIDA :

\begin{abstract}
The bisexual is seen as a creature of uncontrollable impulses (...) whose activities are invariably covert (...) the bisexual is characterized as demonically active, the carrier, the source of spread, the sexually insatiable. At the same time, sexual desire is parceled into two exclusive realms, the homosexual and heterosexual "communities", with the bisexual - understood as a homosexual posing as a heterosexual - acting as the secret conveyor of the diseases of the former to the healthy bodies of the latter (p. 21).
\end{abstract}

Ce passage décrit les bisexuel(le)s comme insatiables, comme des créatures. La bisexualité est le pont entre la communauté hétérosexuelle et celle des homosexuel(le)s. Elle n'a pas une identité propre, mais constitue plutôt une posture, le site qui sert de médiation entre les hommes et les femmes, les hétérosexuel(le)s et les homosexuel(le)s.

Si l'inscription des bisexuel(le)s comme des créatures insatiables efface leurs subjectivités et les établit comme un pont de médiation, en même temps s'inscrit un autre mythe biphobique : les bisexuel(le)s sont perpétuellement confus(es). Comme le note Grover, les identités bisexuelles imaginables, dans les discours dominants sur le SIDA, n'existent pas (ce qui ne nie pas les pratiques bisexuelles) : ce sont des homosexuel(le)s qui se font passer pour les hétérosexuel(le)s. Il y a une position parallèle dans les communautés lesbienne et gaie : on constate 
que la bisexualité n'est qu'une «phase» avant une position de l'homosexualité exclusive, ou bien on constate que les «bisexuel(le)s» sont vraiment des hétérosexuel(le)s ${ }^{7}$. Les opérations des discours binaires exigent qu'on soit hétérosexuel(le) ou homosexuel(le). Il faut choisir un de ces termes. Les bisexuel(le)s - ceux et celles qui ne veulent pas se positionner aux «extrêmes» - sont donc décrit(e)s comme ceux et celles qui ne peuvent pas faire un choix, qui restent donc "perpétuellement confus(es)», toujours en posture.

Ces mythes biphobiques convergent dans des inscriptions «hors texte» des bisexuel(le)s. Cet effacement s'opère de manière variable dans les communautés hétérosexuelles (qui positionnent les bisexuel(le)s comme gai(e)s) et dans les communautés gaies/lesbiennes (qui veulent positionner les bisexuel(le)s comme hétérosexuel(le)s, ou bien qui acceptent leurs identités bisexuelles, mais qui disent : "Allez-y et développez votre propre communauté. Si vous êtes vraiment bisexuel(le), vous n'avez pas le droit de vous insérer dans les communautés lesbiennes/gaies. Si vous voulez le faire, il faut adopter une identité lesbienne / gaie»).

Dans tous les cas, il y a une contradiction : on veut situer les bisexuel(le)s hors des communautés des monosexuel(le)s (soit des hétérosexuel(le)s ou des homosexuel(le)s), mais on reconnaît que les bisexuel(le)s sont toujours-déjà dans ces communautés monosexuelles. Comme l'affirme Carol Queen, parlant aux communautés lesbienne et gaie :

The lesbian and gay movement must come to terms with the position of bisexual people within it because we're already here. It won't help to vote whether or not bisexuals should be let in : we are in (pp. 29-30).

Pourtant, la reconnaissance de cette contradiction - le fait d'écrire les bisexuel(le)s hors texte dans un texte - ne nie pas la vigilance et l'intensité des efforts effectués afin de reconnaître une identité bisexuelle. Amanda Udis-Keller note que ces efforts se produisent de manières différentes chez les hétérosexuel(le)s et les homosexuel(le)s:

(...) both the lesbian and gay and the heterosexual reactions to bisexuals centre not around bisexuality as such, but around homosexuality. The heterosexual reaction to the bisexual is "I'm not gay." The lesbian and gay reaction to the bisexual is "I am gay" (p. 356).

Udis-Keller continue en disant que si on situe une identité bisexuelle comme possible et imaginable, si on réclame la 
bisexualité comme un choix, on va provoquer une crise du sens, «crises of meaning in American culture» (p. 356). De cette façon, Udis-Keller situe la biphobie - et non la bisexualité comme une «crise du sens». Situer la bisexualité comme une identité possible, c'est produire des stratégies vigilantes qui essaient de l'écrire dans le texte.

Le film Basic Instinct est un exemple extraordinaire de cette «crise du sens». En inscrivant les personnages principaux du film en tant que femmes bisexuelles, Basic Instinct reprend les mythes classiques de la bisexualité : les bisexuel(le)s sont obsédé(e)s par le sexe et ils / elles refusent le choix entre l'homosexualité et l'hétérosexualité parce qu'ils / elles sont perpétuellement confus(es). À cet égard, l'articulation de la bisexualité est suivie immédiatement de sa négation. Son énonciation, dans la rubrique hollywoodienne, est associée à la biphobie comme «crise du sens». On accomplit cette inscription biphobique simultanément aux deux niveaux du film : celui de la narration et celui de la sémiotique.

\section{L'inscription narrative de la biphobie : occasions d'obsession}

La tension produite par des personnages qui sont des femmes bisexuelles 8 , présentées comme perpétuellement confuses et obsédées, est évidente dans la structure narrative du film. Par exemple, vers le début du film, les policiers interrogent Catherine à propos du meurtre d'un homme : Johnny Boz. On lui demande si elle avait eu une relation émotive avec lui et elle corrige aussitôt : "I wasn't dating him; I was fucking him». Même si Catherine avait eu des relations sexuelles avec cet homme pendant un an et demie, elle refuse de les définir en termes d'une relation qui implique la tendresse, l'intimité, l'émotion. La manière dont Catherine dit ces mots : «I was fucking him» - voix monotone, sans inflexion - renforce l'idée que les bisexuelles peuvent avoir des relations sexuelles avec n'importe qui, mais pas de véritables relations intimes. Lors de l'entretien avec les policiers, on demande à Catherine si elle regrette le fait que Johnny soit mort. Elle répond : «Yeah, I liked fucking him». L'entretien se poursuit :

POLICIER. - You didn't feel anything for him?

CATHERINE.- In the beginning, no. Then I liked what he did for me.

POLICIER. - That's pretty cold.

CATHERINE. - I'm a writer. I use people for what I write. Let the world beware. 
Cette froideur de Catherine continue pendant tout le film, notamment quand elle dit à Nick : "You won't learn anything I don't want you to know». Plus tard, elle parle de l'histoire de son roman, sorte de métaphore de sa vie (sa condition bisexuelle):

CATHERINE. - Somebody has to die.

NICK. - Why?

CATHERINE. - Somebody always does.

Et, vers la fin du film, Nick dit à Catherine : «Hi. I missed you». Elle répond tout simplement : «Huh». Finalement, elle lui dit : «I can't allow myself to care about you. I can't allow myself to care». Cette caractérisation de la bisexualité sans intimité continue quand Catherine perd son amante Roxy. Elle ne pleure pas lorsqu'elle apprend la nouvelle et tout de suite après, elle baise (avec) Nick. Certes, Catherine représente la bisexuelle par excellence - celle qui n'est pas capable d'émotions, et qui vise à satisfaire ses intérêts sexuels (rappelons le mythe biphobique que les bisexuelles sont insatiables sexuellement).

La représentation de la bisexualité associée à la confusion, dans Basic Instinct, ne se limite pas à l'absence d'émotion. Prenons l'exemple d'Élisabeth, une femme qui était obsédée par Catherine, et qui a changé son look afin d'imiter l'apparence de cette dernière. Le film nous raconte que cette obsession était si accentuée qu'il fallut que Catherine se plaigne d'Élisabeth. Mais quand Élisabeth parle de cet événement à Nick, elle renverse les rôles : elle dit que c'est Catherine qui était obsédée par elle - un renversement qui suggère deux choses à la fois : les bisexuelles ne peuvent pas se connaître; les bisexuelles mentent. De plus, au moment où elle admet avoir baisé avec Catherine, elle nie son identité bisexuelle - ou même la possibilité d'une identité bisexuelle. Elle parle à Nick, son amant (hétérosexuel) : «I was just a kid... I was experimenting... It was just one time... What was I supposed to say?» Elle ajoute au policier : «I'm not gay but I fucked your suspect... I was embarrassed. It was the only time I was with a woman». Ces événements confirment l'impossibilité de faire confiance dans les bisexuelles, qui mentent habituellement. Ainsi Élisabeth est une bisexuelle qui réclame son identité hétérosexuelle pour qu'elle puisse baiser avec Nick, un (autre) hétérosexuel.

Le film présente une équation sans équivoque entre la bisexualité et une tendance obsessive. Reprenons l'exemple d'Élisabeth : elle était complètement, absolument obsédée par 
Catherine - jusqu'à un point dangereux. Il faut noter l'inscription du mythe biphobique que les bisexuelles, surtout à l'époque du SIDA, sont toujours des personnes dangereuses. Le fait qu'elle se soit déguisée, présentée en Catherine suggère une obsession qui désagrège les frontières entre le «je» et «l'autre». Le fait qu'Élisabeth renverse les faits quant aux événements vécus avec Catherine (en disant que c'était Catherine qui était obsédée) fait sens ici : si les bisexuelles sont par définition obsédées, si l'obsession trouble les distinctions entre le «je» et «l'autre», si les bisexuelles sont toujours-déjà perpétuellement confuses, et si elles mentent toujours, alors il s'avère presque impossible pour les bisexuelles de se connaître.

La dissolution des frontières entre le «je» et le «tu» est évidente aussi avec Catherine. En parlant avec Nick, elle lui dit : «Pretty soon I'll know you better than you know yourself». Basic Instinct continue, en suggérant que si on ne se connaît pas, si on ne peut pas faire la distinction entre le «moi» et le «tu», il est impossible d'avoir une relation émotive, intime, et positive. Autrement dit, le film développe ses propres mythes biphobiques de l'obsession bisexuelle afin de dire deux choses reliées : si vous n'êtes pas bisexuel(le), il ne faut pas avoir une relation avec les bisexuel(le)s puisqu'ils / elles ne se connaissent pas; si vous clamez une identité bisexuelle, vous mentez. Parce que les bisexuelles ne peuvent pas se connaître, une vraie identité bisexuelle apparaît inimaginable.

L'inscription des bisexuelles comme personnes obsédées est évidente avec le personnage de Roxy. Amante de Catherine, elle devient très jalouse aussitôt que celle-ci commence à baiser (avec) Nick. Certes, elle essaie de le tuer avec son auto, mais elle a un accident et se tue elle-même. Pendant la chasse, on pense que c'est Catherine qui joue avec Nick, que c'est Catherine qui le tourmente. Quand on découvre que c'était en «réalité» Roxy, une interprétation surgit au niveau de la narration et l'inscription de la biphobie : les bisexuelles étant en général obsédées et ne pouvant se connaître, on ne peut pas, non plus, les connaître. Dès qu'on commence à penser que c'est Catherine, on découvre que c'est Roxy. On répercute cette substitution des bisexuelles sur la dynamique établie entre Catherine et Élisabeth: pendant le déroulement de la narration, on ne sait pas si la personne qui tue ces hommes est Catherine, Élisabeth, ou bien Élisabeth qui se présente en tant que Catherine. La dissolution des frontières entre le «je» et le «tu», inscrite dans une sphère d'obsession, engendre une substitution perpétuelle des personnages et des identités. Est-ce qu'on regarde Catherine ou 
Roxy? Est-ce que c'est Élisabeth ou Catherine qui est coupable? Peu importe, c'est la bisexualité qui provoque cette ambiguïté, cette crise du sens qui engendre la substitution interminable. À cet égard, Basic Instinct rend l'identité bisexuelle, encore une fois, impossible. En même temps, le film déplace la crise du sens de sa biphobie - son impossibilité de concevoir une identité bisexuelle au-delà de ses mythes et stéréotypes - sur la bisexualité.

La structure narrative de Basic Instinct emploie les mythes de la bisexualité et nie l'éventualité de celle-ci. L'écriture de la bisexualité se fait hors texte. Ainsi on inscrit non pas les instincts de base, mais la biphobie de base.

\section{Sémiotique de base, biphobie de base : la crise du «réel»}

La biphobie de Basic Instinct s'inscrit non seulement dans la structure narrative du film, mais aussi dans sa composition sémiotique. Considérons comment on présente les signes de la vie quotidienne dans le film et comment ces signes sont pervertis dans les mains des bisexuelles. Vers la fin du film, on découvre qu'Élisabeth était vraiment obsédée par Catherine et que cette obsession se rattache au passé, à cause des photographies et articles conservés dans un tiroir chez Élizabeth. Si ces documents signifient le souvenir, il est toutefois évident qu'ils sont là à cause de l'obsession (présente). Élizabeth lit les articles, elle regarde les photographies, mais ne s'arrête pas là.

Charles Sauniers Peirce donne une des définitions classiques du signe :

A sign, or representamen, is something which stands to somebody for something in some respect or capacity (...). It stands for that object, not in all respects, but in reference to a sort of idea (...) (cité dans De Lauretis, p. 172).

Les photographies représentent une empreinte d'événements d'une époque historique. Mais dans Basic Instinct elles ne représentent pas seulement les événements du passé, mais l'obsession d'Élizabeth à l'égard de Catherine - une obsession qui existe aujourd'hui. Les signes sont alors pervertis dans deux sens : une incapacité de les interpréter d'une manière fidèle et "correcte», et une perte de distinction entre le passé et le présent.

Cette construction de la perversion des signes - surtout la perversion des articles et documents historiques - est importante pour l'intrigue du film et donc pour l'inscription de sa biphobie. La présentation d'Élizabeth, montrant que son obsession se construit par la perversion des signes, entraîne une 
nouvelle façon de voir Catherine, puisqu'elle a beaucoup d'articles de journaux à propos de Nick et possède les reportages sur la façon dont il a tué deux touristes. De plus, elle connaît très bien ses habitudes personnelles - le fait qu'il fume, par exemple. Catherine dit que ces articles trouvent justification dans sa recherche pour son prochain roman, dont le personnage principal est constitué à partir de la vie de Nick. Mais dans ce contexte de perversion des signes de la vie quotidienne, on se demande si ces articles ne sont pas le site de l'obsession de Catherine.

La perversion des signes par les bisexuelles se manifeste surtout, à travers le film, dans la présentation des signes des meurtres. Hazel, par exemple, a tué sa famille avec un couteau qui était un cadeau de mariage. D'une manière parallèle, Roxy a pris le rasoir de son père afin de tuer ses deux frères. Et la meutrière-bisexuelle (est-ce Catherine ou Élisabeth?) prend un pic à glace. Tous ces objets sont des signes de la vie quotidienne, mais qui sont pervertis par les bisexuelles, dans Basic Instinct, afin d'atteindre un but : la mort. Chaque fois qu'une bisexuelle prend un signe de la vie quotidienne, elle le corrompt, elle détruit l'«ordre naturel», elle remplace la vie (quotidienne) par la mort.

L'équation bisexualité et mort est profondément inscrite à travers le film. Lorsque Nick débute ses relations sexuelles avec Catherine, par exemple, il dit à son ami Gus qu'il a utilisé un condom. Il faut lire cette remarque non pas comme un endossement positif de l'usage des condoms (même si ces références se trouvent peu souvent dans le cinéma hollywoodien), mais plutôt dans son rapport aux mythes biphobiques selon lesquels les bisexuelles sont dangereuses, surtout à l'époque du $\mathrm{SIDA}^{9}$. Si les bisexuelles n'existent pas si elles cachent leurs vraies identités sexuelles (homosexuelles ou hétérosexuelles) - et si, par ces mensonges, elles sont responsables de l'introduction du virus qui peut mener au SIDA dans la "population générale» (les hétérosexuel(le)s), une relation avec l'une d'elle constitue un enchevêtrement avec la mort. À cet égard, la déclaration de Nick qu'il a utilisé un condom est importante, parce que l'objet représente une barrière - littéralement et sémiotiquement - entre Nick et Catherine, l'hétérosexualité et la bisexualité, le corps pur et le corps infecté. Le condom n'est pas ici un signe d'une attitude ouverte par rapport à la sexualité, mais un signe qui délimite les conditions de la sexualité. Si vous allez baiser (avec) une bisexuelle, dit Nick, il faut se méfier. Le condom est ainsi un signe de ce refus 
de la bisexualité10. La construction sémiotique du film suggère que les bisexuelles rompent la stabilité de l'ordre naturel, de la vie quotidienne. On les situe, donc, hors du quotidien et hors de l'«ordre naturel», la famille et l'hétérosexualité. Ce n'est pas une coïncidence si Hazel a utilisé le couteau qu'elle a reçu pour commencer sa famille afin de la détruire, ni que Roxy ait tué ses frères avec le rasoir de son père. Quand les policiers discutent de ces deux événements, on comprend non seulement que les bisexuelles pervertissent les signes de la vie quotidienne, mais aussi qu'elles ne se connaissent pas et que ce manque de connaissance de soi est à la fois dangereux et une substitution perpétuelle (des parallèles s' établissent entre le cas de Hazel et celui de Roxy). A propos de Roxy, un policier dit :

She said she didn't know herself...Did it [a tué ses frères] on impulse. The razor just happened to be there... Just like old Hazel Dobkins fixed her whole family. Except Roxy didn't use a wedding present, she used her daddy's razor.

La constitution de la bisexualité déstabilisée est donc le site de la biphobie («une crise du sens») de Basic Instinct. Affirmant que les bisexuelles ne peuvent pas s'adapter à la vie quotidienne, le film soulève la notion de l'instinct. On constate que les instincts des bisexuelles les mène à tuer. On dit que les bisexuelles sont obsédées, et qu'à cause de leurs obsessions, elles ne sont pas capables de «négocier» le quotidien. Cette tautologie est à la base de la biphobie du film. Les bisexuelles sont toujours-déjà obsédées.

Au niveau de la théorie sémiotique, Basic Instinct nous révèle une élaboration importante de la définition classique du signe. Un signe représente quelque chose, mais dans le contexte d'un système de signification en particulier. Autrement dit, la circulation des signes définit ces derniers, leur donne leur valeur. Comme le disent Robert Hodge et Gunter Kress :

Signs and texts are always socially produced. The structure of signifiers is itself the result of prior social processes of negotiation and contestation, so that the relation of signifiers to signifieds is not arbitrary but signifies the state of social relations at a particular time (p. 229).

Le fait que les bisexuelles ne peuvent pas disposer des signes selon le mode de la culture dominante les inscrit en rupture dans cette circulation ${ }^{11}$. Basic Instinct figure la subjectivité lesbienne / gaie stabilisée, dans le système des signes, dans sa circulation. En racontant qu'elle a baisé avec Catherine, Élisabeth, on l'a vu, nie son identité bisexuelle, même si l'histoire du film nous 
apprend qu'Élisabeth avait au moins une autre amante. Le fait qu'elle énonce le mot (en anglais) "gay» («What was I supposed to say? I'm not gay but I fucked your suspect...») indique que l'homosexualité est une identité possible pour Élizabeth, tandis que la bisexualité ne peut pas s'énoncer. La bisexualité demeure une condition impossible.

Selon Basic Instinct, on peut énoncer les identités monosexuelles - soit une identité hétérosexuelle ou homosexuelle. Mais la bisexualité n'est jamais traitée de front, ne s'articule point. Ce fait suggère que les hétérosexuel(le)s ainsi que les homosexuel(le)s peuvent s'exprimer, mais non les bisexuelles.

Basic Instinct révèle non seulement qu'un signe est quelque chose qui représente autre chose, ni qu'un signe obtient sa valeur dans sa circulation. Il montre comment les signes se présentent en tant que naturels, qu'ils ne font pas que représenter quelque chose d'autre, mais deviennent $c e$ quelque chose d'autre. Ce point est fondamental pour le développement d'une analyse sémiotique critique : la prolifération des signes en tant qu'entités naturelles obscurcit la sémiose qui a lieu.

Cet effacement transparaît souvent dans la culture occidentale. Considérons, par exemple, les drapeaux en tant que signes. Ils deviennent une représentation d'un pays, et plus spécifiquement de sa grandeur. Mais aussitôt que les signes deviennent sacrés, c'est-à-dire naturels, cette fonction symbolique est éludée. Ainsi en Allemagne en 1933 et aux États-Unis en 1989, il y avait une législation qui interdisait de brûler les drapeaux. La distinction entre le drapeau (la toile, les couleurs) et ce qu'il représente (le pays, sa grandeur) est alors effacée. On ne peut pas détruire le drapeau précisément parce que le drapeau ne représente pas seulement le pays, mais est devenu le pays.

Basic Instinct ne parle plus des rasoirs, des couteaux ou des pics à glace par rapport à leurs fonctions quotidiennes et / ou utilitaires. Il y a glissement entre le «naturel» et la «sémiotique» qui constitue un déplacement.

D'une certaine manière, Basic Instinct (comme la culture occidentale) ne dit rien à propos des signes. On les présente comme prédiscursifs, présémiotiques, absoluments réels. À cet égard, la crise du film se déroule autour du réel, ou plus spécifiquement autour de ce qu'on devrait prendre pour le réel. $\mathrm{Si}$ on admet la nature sémiotique du quotidien, il faut admettre aussi qu'on prend le quotidien pour le réel à travers les opérations de la sémiotique. L'élision de la sémiotique situe la bisexualité comme une rupture du réel, et confirme le mythe 
biphobique déjà inscrit dans la structure narrative que les bisexuelles sont obsédées. On parle du réel de façon conservatrice et la bisexualité est positionnée comme une irruption au sein de ce réel. Le titre du film, Basic Instinct, résume très bien toute la problématique.

Citons à nouveau Hodge et Kress :

Social semiotics treats all semiotic acts and processes as social acts and processes. What is always at issue in social processes is the definition of social participants, relations, structures, processes, in terms of solidarity or in terms of power. Semiotic processes are means whereby these can be tested, reaffirmed, altered..."Truth" and "reality" are therefore categories, from a semiotic point of view, which mark agreement over or challenge to the temporary state of the semiotic system (p. 122).

Du point de vue de la sémiotique sociale, il faut contextualiser Basic Instinct en tant que lieu qui essaie de se construire comme «réel» (la vérité), mais qui ne demeure que sémiotique. Le film est donc un lieu qui marque l'inscription de la biphobie, mais qui essaie de la présenter en tant que «naturelle». Voilà le motif du déplacement de la biphobie sur la bisexualité.

\section{Le déplacement de la biphobie sur la bisexualité}

Basic Instinct est un film complexe dans la mesure où on évacue sa nature sémiotique et on reconfirme l'inscription des mythes biphobiques. Une analyse de sa narration démontre que le film présente les bisexuelles comme toujours-déjà obsédées, des personnes qui ne peuvent jamais se connaître. Sa composition sémiotique va plus loin : elle présente ces obsessions comme la condition naturelle de la bisexualité. Si la biphobie est une crise du sens, c'est une crise à la fois narrative et sémiotique. Précisément pour ces raisons, on a besoin d'une analyse sociosémiotique pour faire sens de sa biphobie.

Invoquer la sémiotique met le discours sur le réel - l'instinct - en question. Cette analyse exige une interrogation de la nature construite des significations sociales, et des fonctions normatives des signes et des systèmes de signes. Une analyse sémiotique de Basic Instinct met l'accent sur la prolifération de sa biphobie - comment on écrit le «réel» afin d'écrire les bisexuelles hors texte, et comment cette écriture évacue la nature sémiotique du réel. L'accent sur la sémiotique rend surtout compte du déplacement de la biphobie sur la bisexualité12. Si la bisexualité est caractérisée par des contradictions et des ambiguïtés définitives, une telle analyse est indispensable. Il faut, de plus, analyser ce déplacement si on veut concevoir la possibilité d'une identité bisexuelle au-delà de la biphobie. 
Il faut situer es énoncés de Basic Instinct en tant que crise du sens. Cette manière de procéder évite le projet d'appropriation des lesbiennes et gais qui luttent contre Basic Instinct, interprété comme «homophobique»13. Et, ce qui est peut-être le plus important, une telle interprétation essaie de recontextualiser les termes du débat. On ne discute plus la bisexualité par rapport au réel, on souligne la contradiction (écrire la bisexualité hors texte dans un texte) et on analyse la biphobie comme un symptôme et une articulation de cette contradiction, cette crise du sens. Il s'avère alors encore pensable de concevoir une identité bisexuelle possible et positive.

\section{Université du Québec à Montréal}

\section{NOTES}

1 Comme Fuss, Judith Butler dit que la bisexualité est impossible.

2 "Blatantly Bisexual? Incoherence, Visibility, and Resistance in the Construction of Bisexual Identities." Communication au collège Swarthmore, IV Sager Symposium, 28 mars, 1992. Mes propres réflexions dans ce domaine doivent beaucoup à Michael Du Plessis.

3 Comme du Plessis le note, cette référence traite le mythe de la division de l'hermaphrodite dans deux sexes. Du Plessis continue en disant que " 'psychical hermaphroditism' and 'anatomical [hermaphroditism]' stand as signs in Freud's work for what he keeps on presenting as an originary and original bisexuality. The hermaphrodite, cut up or whole, shadows most of the Three Essays" (p. 3).

4 Il y a plusieurs références à ce phénomène. Prenons, par exemple, le travail de Barbara Havercroft, qui fait une analyse stimulante de l'androgyne dans un roman de Robbe-Grillet. Elle dit que l'androgyne traite les questions du gender, mais termine son article en faisant recours à la sexualité. Elle écrit: "a plurality of 'persons' of indefinite gender and roles, by means of deictic dilemmas that cannot be resolved (...) enact Cixous decription of bisexuality in writing, or a writing which itself could be characterized as bisexual" (p. 54).

5 Voir le travail de Gayle Rubin (1984), qui fait la distinction entre la sexualité et le gender.

6 Le terme «biphobique», qui dérive de la «biphobie», suit la précision du terme «homophobie». "L'homophobie» désigne la peur et / ou la haine irrationelles des homosexuel(le)s. «Biphobie», donc, se définit comme la peur et / ou la haine irrationelles des bisexuel(le)s. Pourtant, je veux développer cette notion de la biphobie, en comprenant que la biphobie essaie de nier la possibilité elle-même d'une identité bisexuelle.

7 Voir la revue Anything That Moves et le livre Bi Any Other Name: Bisexual People Speak Out pour plus de documentation et d'analyse théorique sur les opérations de ces discours binaires qui exigent qu'on soit hétérosexuel(le) ou homosexuel(le). Voir aussi le débat sur la bisexualité dans OUT / LOOK 16 (printemps 1992). L'article de Ara Wilson est un excellent exemple d'explication de la façon dont on considère les bisexuel(le)s comme des hétérosexuel(le)s. Elle conclut son article en écrivant : "While the 
movement for bisexual recognition undoubtable offers a liberating framework for some men and women, some lesbians and gay men remain unmoved by heterosexual transgressions into our entrenched, yet fun, little world».

8 Je parle des «bisexuelles» dans mon analyse du film puisque le film ne présente pas d'hommes bisexuels.

9 Voir du Plessis, op. cit., pour une analyse de la rencontre de la bisexualité féminine et du SIDA dans Basic Instinct.

10 Il y a des inscriptions contradictoires reliées au gender qui sont évidentes ici. Quand Catherine (une femme) dit qu'elle a des relations avec des hommes qui ne sont que sexuelles, elle est nommée «froide». Par contre, quand Nick (un homme) parle du condom et définit sa relation avec Catherine seulement au plan sexuel, on le considère responsable et libéré.

11 Cette conceptualisation de la bisexualité comme rupture provient du travail de Michael du Plessis.

12 À cet égard, on ne peut pas parler de la bisexualité en tant que catégorie sémiotique. Le discours de la socio-sémiotique que je propose essaie de faire sens de la construction (sémiotique) qui met la bisexualité elle-même hors texte.

13 Il faut poser la question suivante : si la biphobie se définit par les efforts de nier la bisexualité, la réponse militante des communautés lesbiennes et gaies n'en fait-elle pas partie? Une analyse socio-sémiotique de Basic Instinct est donc utile pour le développement d'une théorie dite queer, ainsi que pour les stratégies militantes.

\section{OUVRAGES CITÉS}

Anything That Moves.1. San Francisco : Bay Area Bisexual Network.

Butler, Judith. Gender Trouble: Feminism and the Subversion of Identity. New York : Routledge, 1990.

Cixous, Hélène. «Le Rire de la méduse». L'Arc 61 (1975) pp. 39-54.

De Lauretis, Teresa. Alice Doesn't: Feminism, Semiotics, Cinema. Bloomington : Indiana University Press, 1984.

Du Plessis, Michael. «Blatantly Bisexual? Incoherence and Visibility and Resistance in the Construction of Bisexual Identities». Communication au IV Sager Symposium, collège Swarthmore, Pennsylvania, 28 mars, 1992.

Freud, Sigmond. Trois essais sur la sexualité. Traduit de l'allemand par Philippe Koeppel. Paris : Gallimard, 1987.

Fuss, Diana. «Inside / Out», in Dans Diana Fuss (direction), Inside / Out: Lesbian Theories, Gay Theories. New York : Routledge, 1991, pp. 112.

Grover, Jan Zita. "AIDS:Keywords», in Douglas Crimp (direction), AIDS : Cultural Analysis, Cultural Activism. Cambridge, MA : MIT Press, 1988, pp. 17-30.

Havercroft, Barbara. «Deictic Dilemmas and Robbe-Grillet's Andro-Djinn». Recherches sémiotiques/Semiotic Inquiry vol. 10, nos 1-2-3 (1990) pp.39-56.

Hodge, Robert et Kress, Gunter. Social Semiotics. Cambridge : Polity Press, 1988. 
Hutchins, Loraine et Kaahumanu, Lani (eds.). Bi Any Other Name : Bisexual People Speak Out. Boston : Alyson, 1991.

Magnan, Nathalie. «Nous sommes tous des psychopathes!». Gai pied 520 (14 mai, 1992) p. 9.

Queen, Carol. «Strangers at Home : Bisexuals in the Queer Movement» OUT / LOOK 16 (printemps 1992) pp. 23, 29-33.

Rubin, Gayle. «Thinking Sex : Notes Towards a Radical Theory of the Politics of Sexuality», in Carol Vance (ed.), Pleasure and Danger : Explorations in Female Sexuality. London, Boston :Routledge, Kegan Paul, 1984, pp. 267-319.

Udis-Keller, Amanda. «Present Tense : Biphobia as a Crisis in Meaning», in Hutchins, Loraine et Kaahumanu, Lani (eds). Bi Any Other Name: Bisexual People Speak Out. Boston : Alyson Publications, 1991, pp. 350-358.

Wilson, Ara. «Just Add Water : Searching for the Bisexual Politic». OUT/LOOK 16 (printemps 1992) pp. 22, 24-28. 